\title{
A Adolescente Grávida: Alguns Indicadores Sociais
}

\author{
The Pregnant Adolescent: Some Social Indicators
}

\begin{abstract}
Marta Edna Holanda Diógenes Yazlle ${ }^{1}$, Maria Célia Mendes ${ }^{1}$, Maristela Carbol Patta ${ }^{1}$, Juan Stuardo Yazlle Rocha ${ }^{2}$, George Dantas de Azevedo ${ }^{1}$, Alessandra Cristina Marcolin ${ }^{1}$
\end{abstract}

\begin{abstract}
RESUM0
Objetivos: conhecer o número e tipo de partos, categoria de internação, ocupação $e$ diagnósticos obstétricos entre adolescentes de 12 a 19 anos no município de Ribeirão Preto, SP, no periodo de janeiro de 1992 a dezembro de 1996.

Métodos: foram analisadas as informações relacionadas à internação, obtidas das folhas de altas hospitalares, no Centro de Processamento de Dados Hospitalares (CPDH). Para processar as informações, foram utilizados o Sistema Epi-Info 6.04a-processador de texto, banco de dados e estatística para epidemiologia, produzido pelo Centers of Disease Control and Prevention (Atlanta, GA, USA) e o Dbase IV. A associação entre as variáveis foi testada pelo $\chi^{2}$ com nível de significância de $5 \%$. Os parâmetros analisados foram: tipo e número de partos, categoria de internação, ocupação e diagnósticos obstétricos.

Resultados: no periodo estudado ocorreram 42.969 partos, dentre os quais 7.134 (16,6\%) corresponderam aos partos de adolescentes. No decorrer dos anos, houve aumento no número de partos nesta faixa etária, passando de 1.225 partos em 1992 para 1.538 em 1996. Foram relatados partos a partir dos 12 anos, havendo elevação gradual deste número, principalmente depois dos 14 anos, quando houve crescimento de 104,2\% para os partos nesta idade, 48,8\% aos 15 anos, $36,1 \%$ aos 16 anos, $14,0 \%$ aos 17 anos, 52,8\% aos 18 anos e, praticamente, não houve aumento entre aquelas com 19 anos de idade. Na categoria de internação do sistema único de saúde (SUS) foi registrado o maior número de partos (5.709); na categoria pré-pagamento ocorreram 1.277 partos e na categoria particular registraram-se 148 partos. Com relação à ocupação, 14,1\% das pacientes pertenciam à população economicamente ativa e $85,8 \%$ estavam fora da população economicamente ativa. A porcentagem de partos normais foi de 59,2\%, de partos fórcipe foi 5,6\% e cesariana foi de $35,2 \%$. Os diagnósticos obstétricos mais freqüentes foram: problemas do feto ou placenta que afetam a conduta materna (7,9\%), desproporção feto-pélvica $(6,0 \%)$, problemas com cavidade amniótica e membranas (5,0\%), hipertensão complicando o parto e puerpério $(3,5 \%)$ e trabalho de parto prematuro ou falso $(3,4 \%)$.

Conclusão: a maioria dos partos foi normal, ocorrendo com mais freqüência no final da adolescência, principalmente entre aquelas pertencentes à categoria de internação SUS. Houve predomínio de adolescentes não inseridas na população economicamente ativa. Por ocasião da resolução da gestação foram diagnosticadas algumas complicações obstétricas.
\end{abstract}

PALAVRAS-CHAVE: Adolescência. Resolução da gravidez. Complicações da gravidez.

${ }^{1}$ Departamento de Ginecologia e Obstetrícia da Faculdade de Medicina de Ribeirão Preto da Universidade de São Paulo, ${ }^{2}$ Departamento de Medicina Social da Faculdade de Medicina de Ribeirão Preto da Universidade de São Paulo. Correspondência:

Marta Edna Holanda Diógenes Yazlle

Depto de Ginecologia e Obstetrícia - Faculdade de Medicina de Ribeirão Preto, USP

Av. Bandeirantes, 3900 - Monte Alegre

14049-900 - Ribeirão Preto - SP

Telefone: (16) 633-0216 Fax: (16) 633-0946
Introdução

Nos tempos atuais, a problemática da gravidez na adolescência vem assumindo proporções significativas ${ }^{1}$. Dessa forma, tem havido motivação e interesse nas repercussões que a maternidade precoce pode acarretar na saúde das adolescentes, bem como na sua educação, inde- 
pendência econômica e relacionamento so$\mathrm{cial}^{2,3}$.

Quanto ao curso da gravidez, alguns estudos têm demonstrado aumento na incidência de intercorrências pré-natais, intraparto, pós-parto e perinatais entre gestantes adolescentes, notadamente a prematuridade ${ }^{4,5}$ e o baixo peso ao nascimento ${ }^{4}$. Existem ainda relatos de aumento da incidência de restrição de crescimento intra-uterino, sofrimento fetal agudo intra-parto, diabetes gestacional e pré-eclâmpsia ${ }^{1,6-8}$, o que poderia concorrer para maior número de operações cesarianas neste grupo populacional. Outros autores têm demonstrado resultados contrários aos citados previamente, sustentando a idéia de que a gravidez é, fisicamente, bem tolerada pelas adolescentes, notadamente entre aquelas que recebem assistência pré-natal precoce e de forma regular ${ }^{2}$.

Outros fatores, além da qualidade da assistência pré-natal, tais como condições sociais e de saúde, podem exercer mais influência sobre os resultados da gravidez do que, simplesmente, a idade precoce da gestante ${ }^{9}$. Apesar da controvérsia acerca dos resultados obstétricos e perinatais, existe um consenso geral de que o estado gravídico e a maternidade interferem, negativamente, sobre o estilo de vida das adolescentes. Mais importante ainda, são as conseqüências desfavoráveis sobre as suas perspectivas de estudo e trabalho, ou seja, crescimento pessoal e profissional ${ }^{10}$.

Assim, é importante conhecer a magnitude do problema em cada região, a fim de que estratégias possam ser adotadas, para minimizar as repercussões desfavoráveis da gravidez na adolescência e, mais ainda, direcionar as ações preventivas relacionadas ao grupo mais vulnerável para engravidar.

Os objetivos deste estudo foram conhecer número e tipo de parto, categoria de internação, ocupação e diagnósticos obstétricos entre adolescentes de 12 a 19 anos no município de Ribeirão Preto, SP.

\section{Pacientes e Métodos}

Por meio do sistema de informações de altas hospitalares do Centro de Processamento de Dados Hospitalares (CPDH), do Departamento de Medicina Social da FMRP-USP, os autores analisaram, retrospectivamente, os dados correspondentes aos partos de adolescentes na faixa etária de 11 a 19 anos, que ocorreram no município de Ribeirão Preto, São Paulo, no período de janeiro de 1992 a dezembro de 1996.

Para processar as informações, foram utili- zados o programa Epi Info 6.04a-processador de texto, banco de dados e estatística para epidemiologia, produzido pelo "Centers of Disease Control and Prevention" (Atlanta, GA, USA) e o Dbase IV. A associação entre as variáveis foi testada pelo $\chi^{2}$ com nível de significância de 5\%.

Os indicadores sociais utilizados foram a categoria de internação e o tipo de ocupação dessas adolescentes. As categorias de internação foram classificadas segundo o seu financiamento em: particular, pré-pagamento e sistema único de saúde (SUS), que representam a posição social das pacientes, de acordo com o trabalho de Rocha et al. ${ }^{11}$.

As ocupações das adolescentes internadas foram codificadas segundo a Classificação Brasileira de Ocupações para o subsistema de informações sobre mortalidade (Ministério da Saúde, 1987). Estas ocupações foram agrupadas em estratos sociais, segundo o modelo utilizado pelo "Registrar General" na Inglaterra ${ }^{12}$, observando alguns critérios utilizados por Singer et al. ${ }^{13}$ na Bahia. Assim, as pacientes foram distribuídas em população economicamente ativa (PEA) e fora da PEA. A classificação fora da PEA corresponde às ocupações como: donas de casa, estudantes, menores, aposentados e desempregados. A classificação PEA compreende: profissional, intermediário, qualificado não manual, qualificado manual, semiqualificado e não qualificado. Segundo Rocha et al. ${ }^{11}$, esta estratificação representa a posição socioeconômica de importância decrescente.

Para avaliação dos diagnósticos obstétricos, utilizaram-se as informações referidas nas folhas de altas hospitalares, de acordo com a Classificação Internacional de Doenças (CID), 9ª revisão (1985).

\section{Resultados}

No período estudado ocorreram no município de Ribeirão Preto 42.969 partos, dos quais 7.134 foram em adolescentes, correspondendo a 16,6 \% do total de partos. No decorrer dos anos observouse aumento do número de partos nesta faixa etária, tendo ocorrido 1.225 partos em 1992 e 1.538 em 1996 (Tabela 1).

Na mesma tabela encontra-se a freqüência dos partos segundo a idade materna. Observamse partos em adolescentes a partir dos 12 anos, havendo elevação gradual deste número com o aumento da idade. Comparando o número de partos em 1996 com os partos de 1992, em cada grupo etário a partir dos 14 anos, observamos crescimento de $104,2 \%$ para os partos nesta idade, $48,8 \%$ 
aos 15 anos, $36,1 \%$ aos 16 anos, $14,0 \%$ aos 17 anos, $52,8 \%$ aos 18 anos e, praticamente, não houve aumento entre as mães com 19 anos de idade. Na faixa de idade entre 17 e 19 anos houve redução de partos de 1996 em relação aos anos anteriores, sem explicação plausível.

Tabela 1 - Freqüência de partos de adolescentes em Ribeirão Preto, SP, de acordo com a idade materna, no período de 1992-1996.

\begin{tabular}{lrrrrrr}
\hline & 1992 & 1993 & 1994 & 1995 & 1996 & Total \\
\hline 12 anos & 0 & 1 & 2 & 1 & 0 & 4 \\
13 anos & 5 & 3 & 6 & 8 & 8 & 30 \\
14 anos & 24 & 30 & 41 & 36 & 49 & 180 \\
15 anos & 82 & 88 & 93 & 122 & 122 & 507 \\
16 anos & 169 & 191 & 192 & 219 & 230 & 1.001 \\
17 anos & 257 & 256 & 269 & 321 & 293 & 1.396 \\
18 anos & 284 & 426 & 388 & 408 & 434 & 1.940 \\
19 anos & 404 & 394 & 440 & 436 & 402 & 2.076 \\
Total & 1.225 & 1.389 & 1.431 & 1.551 & 1.538 & 7.134 \\
\hline
\end{tabular}

Quanto à categoria de internação, observouse que, em 1992, o número de partos na categoria particular foi de 23, na categoria pré-pagamento foi 173 e na categoria SUS foi 1.029. No ano de 1993 , ocorreram 28 partos na categoria particular, 241 partos na categoria pré-pagamento e 1.120 na categoria SUS. Em 1994, nas categorias particular, pré-pagamento e SUS ocorreram 27, 263 e 1.141 partos, respectivamente. Em 1995, ocorreram 34 partos na categoria particular, 300 na prépagamento e 1.217 na categoria SUS. Em 1996, houve 36 partos na categoria particular, 300 na categoria pré-pagamento e 1.202 na categoria SUS. O total de partos ocorridos no período de 1992 a 1996 foi de 7.134 , sendo $148(2,1 \%)$ na categoria particular, 1.277 ( $17,9 \%)$ na categoria pré-pagamento e 5.709 ( 80,0\%) na categoria SUS.

Na Tabela 2 estão distribuídas as gestantes adolescentes de acordo com a idade e a categoria de internação. Verifica-se que na categoria SUS foram encontrados partos a partir de 12 anos, ao passo que nas categorias pré-pagamento e particular a idade mínima das gestantes foi de 13 e 14 anos, respectivamente. Com o aumento da idade o número de partos foi crescente, variando de $0,06 \%$ aos 12 anos a $29,1 \%$ aos 19 anos, destacando-se que dos 15 aos 16 anos houve aumento de 7,1 para $14,2 \%$.

No que se refere a ocupação, 1.005 (14,1\%) adolescentes grávidas já tinham inserção na PEA, $482(6,8 \%)$ eram estudantes, 5.637 (79,0\%) eram do lar, menores e sem ocupação remunerada. Em dez $(0,1 \%)$ pacientes não havia informação sobre a ocupação.

Tabela 2 - Distribuição dos partos de adolescentes por idade materna e categoria de internação hospitalar, em Ribeirão Preto, SP, no período de 1992-1996.

\begin{tabular}{lccrrr}
\hline & Particular & Pré-pagamento & SUS & \multicolumn{2}{c}{ Total } \\
& & & & \multicolumn{1}{c}{ n } & \multicolumn{1}{c}{$\%$} \\
\hline 12 anos & 0 & 0 & 4 & 4 & 0,06 \\
13 anos & 0 & 5 & 25 & 30 & 0,42 \\
14 anos & 1 & 25 & 154 & 180 & 2,50 \\
15 anos & 4 & 58 & 445 & 507 & 7,10 \\
16 anos & 13 & 168 & 820 & 1.001 & 14,20 \\
17 anos & 28 & 250 & 1.118 & 1.396 & 19,50 \\
18 anos & 45 & 361 & 1.534 & 1.940 & 27,20 \\
19 anos & 57 & 410 & 1.609 & 2.076 & 29,10 \\
Total & 148 & 1.277 & 5.709 & 7.134 & 100,00 \\
\hline
\end{tabular}

Com relação ao tipo de parto, observou-se que, no período avaliado, $4.211(59,2 \%)$ adolescentes tiveram partos normais, $396(5,6 \%)$ tiveram partos fórcipe e $2.502(35,2 \%)$ tiveram cesarianas, de um total de 7.109 partos informados. No decorrer dos anos, o número de partos normais variou de 760 $(62,4 \%)$ em 1992 para 879 (56,7\%) em 1996, o número de parto fórcipe variou de 44 (3,6\%) em 1992 para $133(8,6 \%)$ em 1996 e o de cesariana variou de 404 (34\%) em 1992 para 531 (36,6\%) em 1996. O número total de partos ocorridos no período de 1992 1996 correspondeu a $1.218,1.380,1.417,1.551 \mathrm{e}$ 1.543 em cada ano, respectivamente.

Os diagnósticos obstétricos mais freqüentes, de acordo com a CID (9 ${ }^{a}$ revisão de 1985), foram: problemas do feto ou placenta que afetam a conduta materna, como malformação fetal hereditária ou causada por vírus ou drogas, hemorragia feto-materna, isoimunização Rh ou ABO, sofrimento fetal, morte intra-uterina, crescimento fetal insuficiente ou excessivo e anormalidade placentária (565), desproporção feto-pélvica (426), problemas com a cavidade amniótica e membranas (355), hipertensão complicando o parto e o puerpério (249) e trabalho de parto prematuro ou falso (241) (Tabela 3).

\section{Discussão}

Os dados utilizados no presente estudo foram obtidos a partir de bases populacionais, isto é, abrangem todos os hospitais e as hospitalizações da cidade e região de Ribeirão Preto, onde há cobertura total da assistência ao parto ${ }^{1}$. A propor- 
ção de partos entre adolescentes em relação ao total de partos, no período de janeiro de 1992 a dezembro de 1996, foi de 16,6\%. Quando avaliamos o crescimento do número de partos segundo a idade materna com o decorrer do tempo, de 1992 para 1996, observamos maior aumento percentual aos 14 anos (104,2\%) diminuindo, gradualmente, até os 17 anos (14\%). Aos 18 anos houve nova elevação $(52,8 \%)$ que pode ser explicada pela freqüência dos casamentos precoces no nosso meio. No Brasil, Prado ${ }^{14}$ referiu incidência correspondente a $21 \%$ e Costa et al. ${ }^{15}$, a 21,6\%. Nos Estados Unidos, segundo Martin et al. ${ }^{16}$, houve redução das taxas de nascimento na população de adolescentes a partir de 1991.

Tabela 3 - Distribuição dos diagnósticos obstétricos de adolescentes admitidas para resolução da gravidez em Ribeirão Preto, no período de 1992-1996.

\begin{tabular}{|c|c|c|}
\hline \multirow[t]{2}{*}{ Diagnósticos (CID*) } & \multicolumn{2}{|c|}{ Total } \\
\hline & $\mathbf{n}$ & $\%$ \\
\hline Parto normal (650) & 3.698 & 51,8 \\
\hline $\begin{array}{l}\text { Complicações do trabalho de parto e parto } \\
\text { não classificadas em outra parte (669) }\end{array}$ & 888 & 12,4 \\
\hline $\begin{array}{l}\text { Problemas fetais ou da placenta afetando a } \\
\text { conduta em relação a mãe (656) }\end{array}$ & 565 & 7,9 \\
\hline Desproporção feto-pélvica (653) & 426 & 6,0 \\
\hline $\begin{array}{l}\text { Problemas com a cavidade amniótica e } \\
\text { Membranas (658) }\end{array}$ & 355 & 5,0 \\
\hline $\begin{array}{l}\text { Hipertensão complicando o parto e } \\
\text { Puerpério (642) }\end{array}$ & 249 & 3,5 \\
\hline Trabalho de parto prematuro ou falso (644) & 241 & 3,4 \\
\hline
\end{tabular}

Muito se tem discutido sobre a relação entre a incidência de gravidez na adolescência e as condições sociais e econômicas desta população. Segundo Guijarro et al. ${ }^{17}$, adolescentes grávidas relataram menor comunicação intrafamiliar, menor satisfação pessoal e maiores dificuldades escolares e econômicas. Outros sugerem que as adolescentes que não engravidam apresentam maior religiosidade, pais com melhor nível de educação e ambos os pais trabalhando fora de casa ${ }^{11,17}$.

A falta de recurso social, de acordo com Lesser e Escoto-Lloyd ${ }^{3}$, contribui para os problemas de saúde das adolescentes grávidas e das mães jovens. A sobrecarga de responsabilidade, decorrente da chegada do filho, ocasiona modificação radical no estilo de vida das adolescentes. Além disso, a discriminação ainda hoje existente, por parte da sociedade e até da própria família, pode determinar sérias conseqüências de ordem psicológica e social.

No estudo em questão, observou-se maior porcentagem dos partos das adolescentes na categoria de internação SUS, quando comparado às outras categorias (pré-pagamento e particular), semelhante ao que acontece com a população geral de mulheres no município de Ribeirão Preto ${ }^{18}$. Além disso, no decorrer dos anos, houve elevação do número de partos de adolescentes em todas as categorias de internação. Isto sugere que algumas medidas de prevenção de gravidez na adolescência devam ser adotadas, principalmente, dirigidas às adolescentes de nível socioeconômico baixo, o que corresponde, neste município, à população atendida pelo SUS ${ }^{9}$.

Sabe-se que aproximadamente $30 \%$ das adolescentes grávidas abandonam a escola, e na maioria dos casos, o retorno ao estudo se dá em menores proporções ${ }^{19,20}$. Entretanto, a evasão escolar, às vezes, precede a gravidez, sendo inclusive uma condição de risco para engravidar ${ }^{10}$. Em vista disso, aquelas que abandonam seus estudos não se profissionalizam e, conseqüentemente, terão trabalho mal remunerado no futuro.

No presente estudo, 85\% das adolescentes foram classificadas como fora da PEA, portanto não tinham trabalho remunerado, sendo a maioria pertencente à categoria do lar. A idade é um dos fatores que determinam que essas pacientes estejam fora da PEA, pois nesta fase da vida elas ainda não possuem capacitação profissional. Com a ocorrência de gravidez neste período, a chance para conseguirem fazer parte da PEA torna-se ainda menor. Se considerarmos que as pacientes pertencentes à categoria SUS são de nível socioeconômico mais baixo, confirmando assim os dados da literatura, há possibilidade de permanecerem em categoria social menos favorecida.

Com relação ao tipo de parto, foi observado freqüência de cesariana correspondente a 35,2\%. A literatura nacional mostra taxa de cesárea elevada na população adulta ${ }^{21,22}$, o que pode refletir na população mais jovem, estando provavelmente relacionada com os fatores que levaram a mudança na prática obstétrica no Brasil. Outro fator que também poderia justificar as taxas elevadas de cesárea no estudo em questão é a presença de expressivo número de intercorrências obstétricas, como: o parto pré-termo, as síndromes hipertensivas e a desproporção feto-pélvica. São intercorrências da gravidez semelhantes àquelas observadas na literatura ${ }^{6,23}$.

Existe controvérsia quanto ao fato de a gravidez na adolescência aumentar o risco de complicações obstétricas, perinatais e neonatais. Alguns autores julgam que há elevação do risco ${ }^{1,6,23}$, ao passo que outros acreditam que o risco dessas 
complicações não aumenta na adolescente, quando comparado à população adulta ${ }^{2}$.

Os resultados apresentados permitem concluir que: houve aumento do número de partos entre adolescentes no município de Ribeirão Preto de janeiro de 1992 a dezembro de 1996; a maioria dos partos foi normal, ocorrendo com mais freqüência no final da adolescência, principalmente entre aquelas pertencentes à categoria de internação do SUS houve predomínio de adolescentes não inseridas na PEA; e por ocasião da resolução da gestação foram diagnosticadas algumas complicações obstétricas; as medidas estratégicas para prevenção da gestação na adolescência devem ser direcionadas, principalmente, à população de baixo nível socioeconômico-cultural.

\section{ABSTRACT}

Purpose: the objective of the present study was to determine the number and type of deliveries, category of admission to the hospital, occupation and obstetrical diagnosis for adolescents from the municipality of Ribeirão Preto, from January 1992 to December 1996.

Methods: the information obtained from hospital discharge forms was analyzed at the Hospital Data Processing Center. The 6.04a-text processor Epi-Info System, a data bank and statistics for epidemiology produced by the Centers of Disease Control and Prevention (Atlanta, GA, USA), and Dbase IV were used to process the information. The association between variables was tested by the $\chi^{2}$ test, with the level of significance set at 5\%. The analyzed parameters were: number and type of delivery, category of hospital admission, occupation and obstetric diagnosis.

Results: a total of 42,969 deliveries occurred during the study period, among which 7,134 (16.6\%) corresponded to adolescent deliveries. An increase in the number of deliveries by girls in this age range occurred over the years, from 1,225 in 1992 to 1,538 in 1996. Deliveries were reported starting from 12 years of age, with a gradual increase in this number, especially after 14 years of age, when a $104.2 \%$ increase occurred for deliveries at this age, followed by $48.8 \%$ at 15 years, $36.1 \%$ at 16 years, $14.0 \%$ at 17 years, $52.8 \%$ at 18 years, and with practically no increase among 19-year-old girls. The highest number of deliveries $(5,709)$ was recorded for the unified health system category of admission, followed by 1,277 deliveries for the prepaid category and 148 deliveries for the private category. With respect to occupation, $14.1 \%$ of the patients belonged to the economically active population, while $85.8 \%$ did not. Of the total deliveries, $59.2 \%$ were normal, $5.6 \%$ were forceps deliveries, and $35.2 \%$ were cesarean sections. The most frequent obstetrical diagnoses were: problems of fetus or placenta affecting maternal management (7.9\%), fetus-pelvis disproportion (6.0\%), problems with the amniotic cavity and membranes (5.0\%), hypertension complicating delivery and puerperium
(3.5\%), and premature or false labor (3.4\%).

Conclusions: most deliveries were normal and occurred more frequently at the end of adolescence, especially among girls belonging to the unified health system. There was a predominance of adolescents not belonging to the economically active population. Some obstetrical complications were diagnosed at the time of resolution of pregnancy.

KEY WORDS: Adolescence. Resolution of pregnancy. Pregnancy complications.

\section{Referências}

1. Barros FC, Vaughan JP, Victora CG, Huttly SR. Epidemic of caesarean sections in Brazil. Lancet 1991; 338:167-9.

2. Lao TT, Ho LF. The obstetric implications of teenage pregnancy. Hum Reprod 1997; 12:2303-5.

3. Lesser J, Escoto-Lloyd S. Health-related problems in a vulnerable population: pregnant teens and adolescent mothers. Nurs Clin North Am 1999; 14:289-99.

4. Fraser AM, Brockert JE, Ward RH. Association of young maternal age with adverse reproductive outcomes. N Engl J Med 1995; 332:1113-7.

5. Jolly MC, Sebire N, Harris J, Robinson S, Regan L. Obstetric risks of pregnancy in women less than 18 years old. Obstet Gynecol 2000; 96:962-6.

6. Orvos H, Nyirati I, Hajdu J, Pal A, Nyari T, Kovacs L. Is adolescent pregnancy associated with adverse perinatal outcome? J Perinatal Med 1999; 27:199203.

7. Abu-Heija A, Ali AM, Al-Dakheil S. Obstetrics and perinatal outcome of adolescent nulliparous pregnant women. Gynecol Obstet Invest 2002; 53:902.

8. Eure CR, Lindsay MK, Graves WL. Risk of adverse pregnancy outcomes in young adolescent parturients in an inner-city hospital. Am J Obstet Gynecol 2002; 186:918-20.

9. Rocha JSY, Simões BJG. Estudo da assistência hospitalar pública e privada em bases populacionais, 1986-1996. Rev Saúde Pública 1999; 33:44-54.

10.Stevens-Simon C, Lowy R. Teenage childbearing. An adaptive strategy for the socioeconomically disadvantaged or a strategy for adapting to socioeconomic disadvantage? Arch Pediatr Adolesc Med 1995; 149:912-5.

11.Rocha JSY, Simões BJG, Guedes GLM. Assistência hospitalar como indicador da desigualdade social. Rev Saúde Pública 1997; 31:479-87. 
12.Townsend $\mathrm{P}$, Davidson $\mathrm{N}$, editors. Inequalities in health: the Black report. $1^{\text {st }}$ ed. London: Penguin Books; 1982.

13.Singer P, Oliveira EM, Luchesi MA, Santos JLF, Nigro S. Demanda por alimentos na área metropolitana de Salvador. $1^{\text {a }}$ ed. São Paulo: CEBRAP/Brasiliense; 1976. (Cad. CEBRAP, 23).

14.Prado LV. Gravidez não planejada. Adolescência 1996; 45:23-4.

15. Costa MCO, Santos CAT, Nascimento Sobrinho CL, et al. Childbirth and live newborns of adolescent and young adult mothers in the municipality of Feira de Santana, Bahia State, Brazil, 1998. Cad Saúde Pública 2002; 18:715-22.

16. Martin JA, Park MM, Sutton PD. Births: preliminary data for 2001. Natl Vital Stat Rep 2002; 50:1-20.

17.Guijarro S, Naranjo J, Padilla M, Gutierez R, Lammers C, Blum RW. Family risk factors associated with adolescent pregnancy: study of a group of adolescent girls and their families in Ecuador. J Adolesc Health 1999; 25:166-72.
18.Yazlle MEHD, Rocha JSY, Mendes MC, Patta MC, Marcolin AC, Azevedo GD. Incidência de cesáreas segundo fonte de financiamento da assistência ao parto. Rev Saúde Pública 2001; 35:202-6.

19.Blum RW, Geer L, Hutton L, et al. The Minnesota adolescent health survey. Implications for physicians. Minn Med 1988; 71:143-5, 149.

20.Upchurch DM, McCarthy J. The timing of a first birth and high school completion. Am Sociol Rev 1990; $55: 224-34$

21.Bozkya $H$, Mocan $H$, Usluca $H$, Beser $E$, Gumustekin D. A retrospective analysis of adolescent pregnancies. Gynecol Obstet Invest 1996; 42:146-50.

22.Gentile FP, Noronha Filho G, Cunha AA. Associação entre a remuneração da assistência ao parto e a prevalência de cesariana em maternidades do Rio de Janeiro: uma revisão da hipótese de Carlos Gentile de Mello. Cad Saúde Pública 1997; 13:221-6.

23.Kumbi S, Isehak A. Obstetric outcome of teenage pregnancy in northwestern Ethiopia. East Afr Med J 1999; 76:138-40.

Recebido em: 25/4/2002 Aceito com modificações em: 27/9/2002

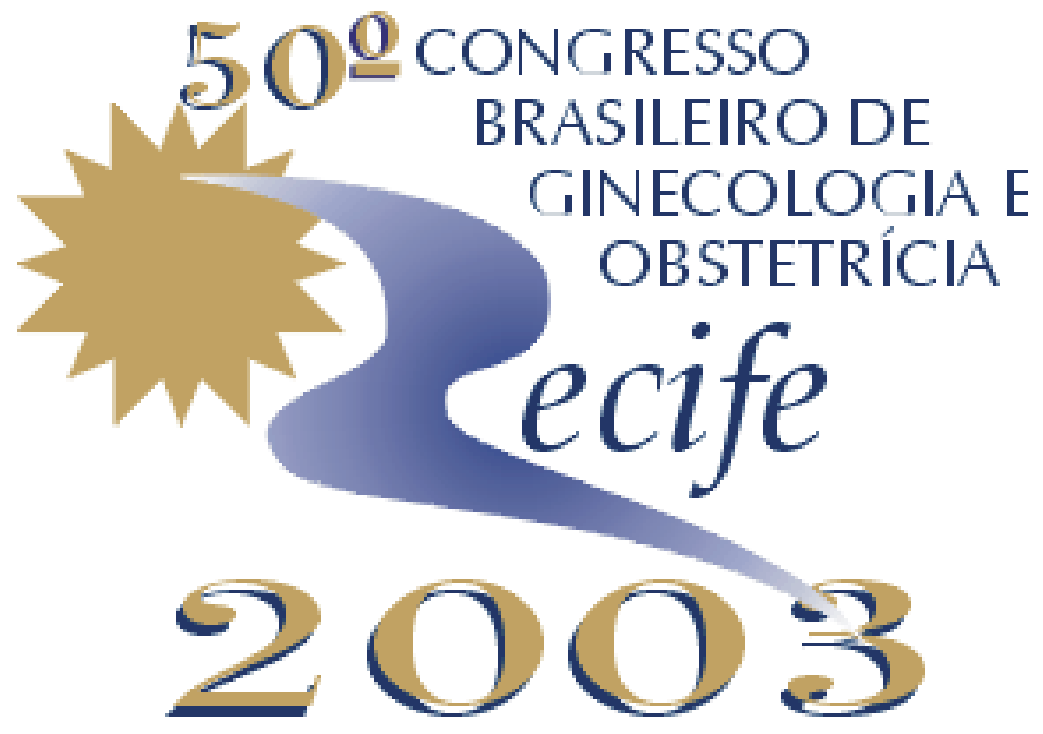

18 a 22 de novembro INFORMAÇOES: Telefone: (81) 3222-5112 E-mail: sogope1@terra.com.br 